\title{
Modelling and control of a medium-duty hybrid electric truck
}

\author{
C.-C. Lin, Z. Filipi, L. Louca, H. Peng*, \\ D. Assanis, J. Stein
}

\begin{abstract}
Automotive Research Center, The University of Michigan
*Corresponding author, G036 Lay Auto Lab, Department of Mechanical Engineering, University of Michigan, Ann Arbor, MI 48109-2133. Email: hpeng@umich.edu.
\end{abstract}

\begin{abstract}
The main contributions of this paper are the development of a forward-looking hybrid vehicle simulation tool, and its application to the design of a power management control algorithm. The hybrid electric vehicle simulation tool (HE-VESIM) was developed at the Automotive Research Center of the University of Michigan to study the potential fuel economy and emission benefits of the parallel hybrid propulsion system for a medium truck. The fundamental architecture of the feed-forward simulation tool and the dynamic equations of its sub-system modules are first described. A power management control algorithm is then designed and evaluated, which is based on mimicking the behaviour of a dynamic-programming optimisation scheme. Simulation results over an urban driving cycle demonstrate that the hybrid control algorithm is able to improve vehicle fuel economy significantly, compared with the original vehicle, powered only by a diesel engine.
\end{abstract}

Keywords: electric vehicles, electric-vehicle simulation, hybrid electric vehicles, hybrid vehicles, vehicle modelling, vehicle simulation.

Reference to this paper should be made as follows: Lin, C-C., Zoran, F., Louca, L., Peng, H., Assanis, D. and Stein, J. (2004) 'Modelling and control of a medium-duty hybrid electric truck', Int. J. of Heavy Vehicle Systems, Vol. 11, Nos. 3/4, pp. 349-370.

\section{Introduction}

Growing environmental concerns, coupled with the complex issue of global crude oil supplies, stimulate research aimed at the development of fuel-efficient vehicles. Recently, advanced diesel engines, fuel cells, and hybrid powertrains have been actively studied as potential technologies for future ground vehicles. Hybrid-electric vehicles (HEV) appear to be one of the most viable technologies with significant potential to reduce fuel consumption within realistic economic, infrastructural and customer acceptance constraints. Dozens of prototype concept hybrid vehicles have been developed. Toyota and Honda have successfully marketed production vehicles and many other major automakers are expected to launch hybrid vehicles in the next 1-5 years. Due to the existence of dual power-sources, the additional design degrees of freedom of HEV 
offer unprecedented possibilities in fuel economy and exhaust emissions, especially when parallel powertrain architecture is employed. However, the complexity of the new vehicle system requires extensive and highly-accurate simulations for proper component sizing, as well as for the development of control algorithms to maximise the potential of these advanced technologies.

While much of the existing literature focuses on passenger cars or SUV/light trucks, the target vehicle for this paper is a Class VI medium-duty truck. There are two reasons for this. First, commercial trucks have a higher per-unit price, and thus it is easier to justify the added cost of hybrid components. Secondly, fuel savings and the trickle-down effect on reducing emissions of these commercial trucks are more significant, because commercial vehicles usually use a lot more fuel over their life compared with individually-owned vehicles.

A significant difference between the simulation model developed in this paper, compared with existing tools such as ADVISOR (Burch et al., 1999), lies in the fact that the HE-VESIM model we developed is a forward-looking model. The backward simulation tools, such as ADVISOR, assume that the driving cycle has been followed accurately, and then compute the upstream variables, such as gas pedal position and fuel consumption. In the forward looking approach, we take driver throttle/brake command to calculate vehicle response, i.e. the flow of information is exactly the same as in the physical system. This difference is important because it enables us to study the effect of different control algorithms under realistic transient conditions. In this paper, the design of the power management control algorithm is presented, which deals with the power split strategy between the internal combustion engine and the electric powertrain (battery/motor), as well as with the gear shifting strategy. We will focus on this higherlevel control design problem, and assume that the servo-loop control algorithms are fast and accurate enough to be ignored.

Most of the existing power management control strategies developed for parallel HEV fall into three categories. The first type applies rule-based, fuzzy logic or neural network techniques for control and estimation algorithm development (Baumann et al., 2000; Farrall et al., 1993). The rules are frequently based on the operation of a single sub-system, e.g. load levelling for improved engine efficiency, charging/discharging level based on battery efficiency, etc. The second type of approach is based on static optimisation methods. For example, to calculate the overall fuel consumption, the electric energy consumed is translated into an equivalent amount of fuel (Kim et al., 1999; Paganelli et al., 2000). The optimisation scheme then identifies the proper split between the two power sources using static efficiency maps of the sub-systems. Due to the pointwise nature of static optimisations, it is relatively easy to extend this scheme to solve the simultaneous fuel economy and emission optimisation problem (Johnson et al., 2000). The basic idea of the third type of HEV control algorithm is similar to that of static optimisation; however, the optimisation is performed for dynamic systems (Brahma et al., 2000; Kang et al., 1999). Further, the optimisation is usually with respect to a time horizon (e.g. a given driving cycle), rather than for a fixed point in time. Usually, the power split algorithm obtained from a dynamic optimisation process will be more accurate under transient conditions. If the dynamic optimisation algorithm is obtained through a computation procedure, such as dynamic programming, that can yield a true optimum, the obtained control law is not implementable due to its preview nature and 
heavy computation requirement. It is, however, a good benchmark based on which the first two types of algorithms can be improved or compared against.

The objective of this paper is to develop an integrated hybrid vehicle simulation tool and use it for the design of power management control algorithms. The basis for our Hybrid Vehicle-Engine SIMulation (HE-VESIM) is the high-fidelity conventional vehicle simulator VESIM previously developed at the University of Michigan (Assanis et al., 2000). VESIM has been validated against measurements for a Class VI truck, and was proven to be a versatile tool for mobility, fuel economy and drivability studies. To construct a hybrid-vehicle simulation program, some of the main modules are modified, e.g. the engine is reduced in size/power, and the electric component models are created and integrated into the system. Our HEV simulation effort focuses on a parallel posttransmission configuration, where the electric motor is mechanically coupled to the output shaft of the transmission. The integrated simulation model is implemented in SIMULINK to allow for easy reconfiguration of the system and selection of proper models depending on specific simulation goals.

The paper is arranged as follows. The configuration of the hybrid electric vehicle system is discussed first. The main simulation modules: diesel engine, drivetrain, vehicle dynamics and electric components, are presented next. Then, a sub-optimal rule-based power management control algorithm is introduced. The complete hybrid vehicle simulation is subsequently used to assess the acceleration ability and the fuel economy of the hybrid vehicle through comparisons with its conventional counterpart. The control strategy is evaluated through simulation predictions of fuel consumption over a driving cycle, followed by the summary and conclusions.

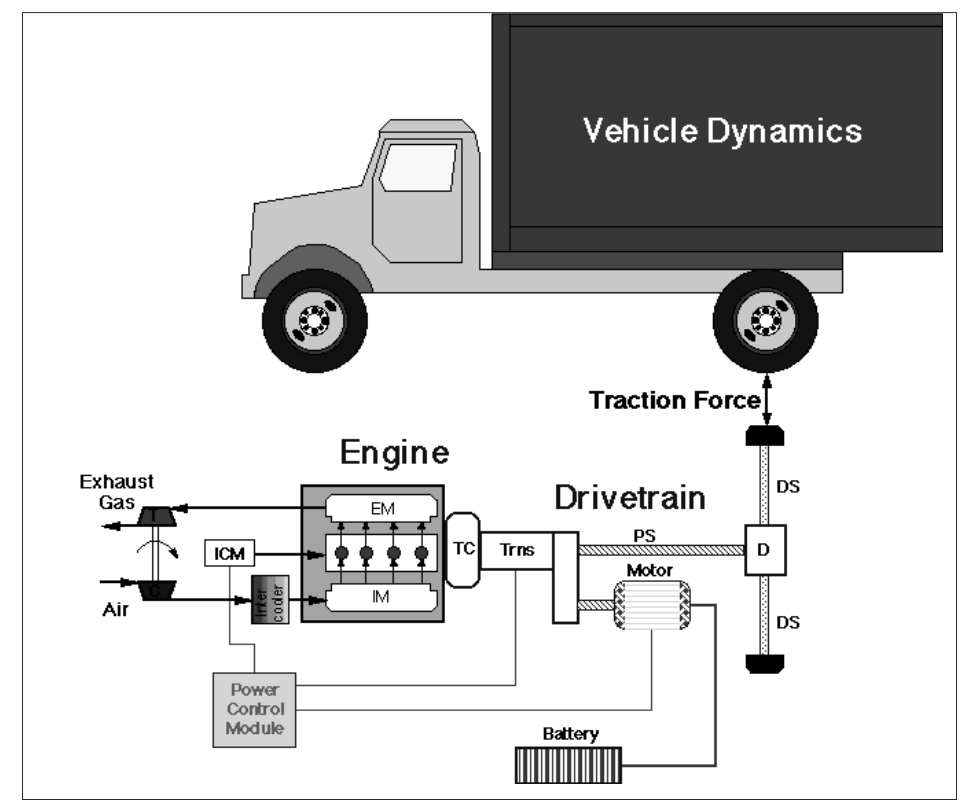

Figure 1 Schematic of the integrated vehicle system. 


\section{Hybrid-electric vehicle system}

The target vehicle considered in this paper is a $4 \times 2$ Class VI truck. The hybrid version of which is assumed to be a parallel hybrid vehicle with a permanent magnet DC brushless motor positioned after the transmission (see Figure 1). The engine is connected to the torque converter (TC), the output shaft of which is then coupled to the transmission (Trns). The electric motor is linked to the propeller shaft (PS), differential (D) and two driveshafts (DS). The motor can be run reversely as a generator, by drawing power from regenerative braking or from the engine.

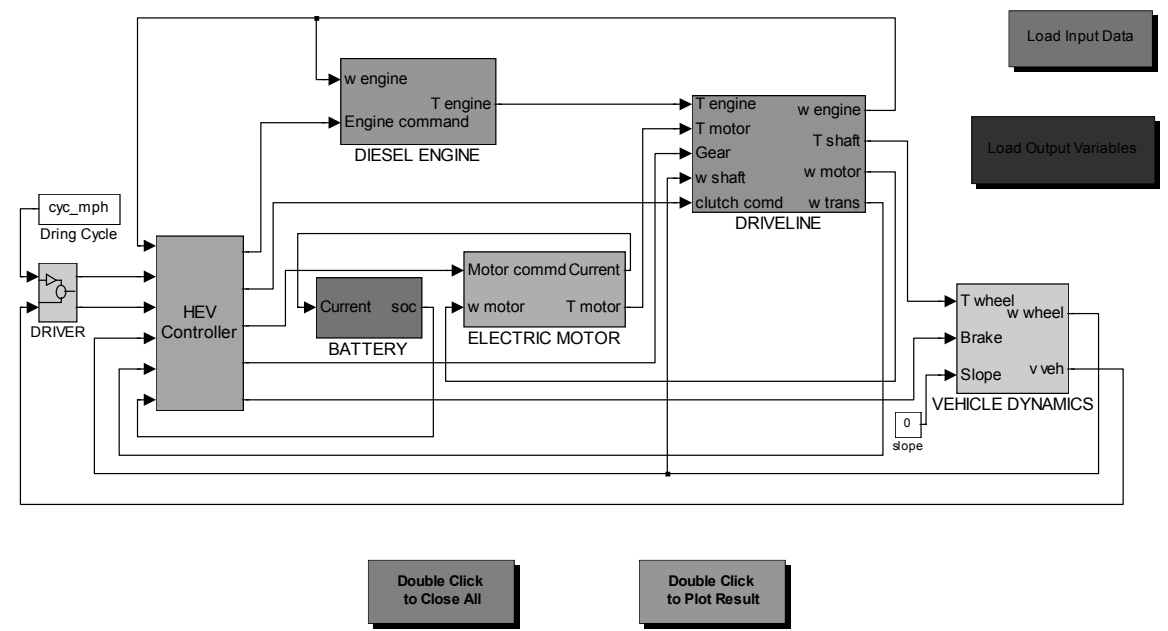

Figure 2 Hybrid-electric vehicle simulation in SIMULINK.

To maintain high modularity, the simulation model is implemented in the MATLAB/SIMULINK environment, as shown in Figure 2. Links between the modules represent the physical variables that define the interaction between the components, such as torque, angular velocity, electrical current or voltage. The HEV controller contains the power management logic and sends control signals (power demand) to the components modules, which are assumed to be achieved accurately. A 'driver' module is designed to follow a prescribed driving cycle, i.e. a speed trajectory specified over time. Other blocks shown in Figure 2 are discussed in the following sub-sections.

\subsection{Engine}

The engine model is derived from the high fidelity, thermodynamic engine system previously developed for the conventional vehicle (Assanis et al., 2000; Assanis et al., 1986). The high fidelity engine model consists of multiple cylinder modules linked with external component modules for manifolds, compressors and turbines, heat exchangers, air filters, and exhaust system elements. To support the computationally intensive simulations over long driving cycles and facilitate easy scaling of the engine (for component sizing), the thermodynamic engine model is replaced by a look-up table that provides brake torque as a function of instantaneous engine speed and fuel injection rate. 
The look-up table is generated by using the validated high fidelity engine system code (Assanis et al., 2000; Assanis et al., 1986), hence it is possible to vary the size of the engine physically, or its design, and have a realistic representation of the effect of the change. For the hybrid truck, the original V8 $7.3 \mathrm{~L}$ diesel engine is downsized by reducing the number of cylinders from 8 to 6 , and hence the displacement to $5.5 \mathrm{~L}$. The turbo-machinery maps are scaled down to match the smaller engine, following the methodology described in (Assanis, et al. 1999). The whole procedure for generating torque look-up tables based on predictions of a validated high fidelity engine system code is illustrated in Figure 3. The specifications of both the V8 engine for the conventional vehicle and the V6 engine for the hybrid application are given in Table 1.

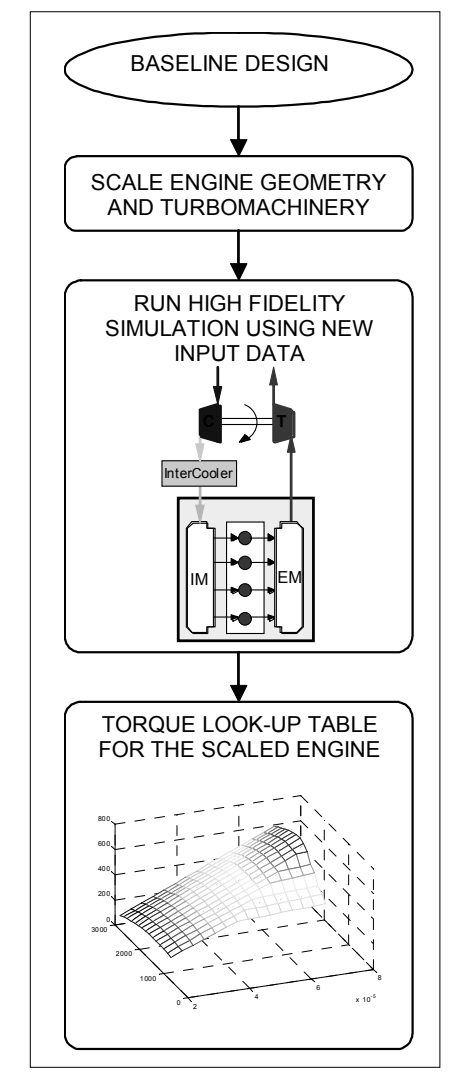

Figure 3 Torque map generation for a scaled engine.

To retain features of the engine system critical for the transient response, the complete fuel control logic is kept unchanged in the look-up table based model. The diesel engine fuel injection controller regulates the mass of fuel injected per cycle based on driver demand supplied by the driver module, environmental conditions and engine speed and boost pressure. The engine speed is the output of the engine dynamics block, and the nominal value of boost pressure is tabulated as a function of speed and load. Hence, the fuel control logic that limits the fuel at low boost is retained in a reasonable way. In addition, a carefully calibrated time delay is built-in to represent the turbo-lag in 
response to rapid increases of engine rack positions. More details about the engine subsystem and the fuel controller are provided in the work by Assanis et al., (2000).

Table 1 Diesel engine specification.

\begin{tabular}{lcc}
\hline & Conventional & Hybrid \\
\hline Configuration & V8, Turbocharged, Intercooled & $\begin{array}{c}\text { V6, Turbocharged, } \\
\text { Intercooled }\end{array}$ \\
Displacement [L] & 7.3 & 5.475 \\
Bore [cm] & 10.44 & 10.44 \\
Stroke [cm] & 10.62 & 10.62 \\
Con. Rod Length [cm] & 18.11 & 18.11 \\
Compression Ratio [-] & 17.4 & 17.4 \\
Rated Power [hp] & $210 @ 2400 \mathrm{rpm}$ & $157 @ 2400 \mathrm{rpm}$ \\
\hline
\end{tabular}

\subsection{Drivetrain}

The driveline module consists of the torque converter, transmission, propshafts, differential, and drive shafts. It provides the connection between the engine, the electric motor and the vehicle body (see Figure 1). The torque converter input shaft connects to the engine flywheel. On the other end, the transmission-output shaft and the electric motor shaft are connected to the propeller shaft, the differential and, via driveshafts, to the two driven wheels. The drivetrain model is constructed using the bond graph modelling language (Karnopp et al., 1990; Rosenberg and Karnopp, 1983) and implemented in the 20SIM software environment (20SIM, 1999). The bond graph language is selected due to its capability of effortless generation of models with different complexity (Louca et al., 1998). The development of the drivetrain model is described in (Louca et al., 2001); its key parameter values are given in Table A1 in the Appendix.

The dynamic behaviour of the drivetrain is represented by ordinary differential equations that describe the kinematic and dynamic behaviour of the real system. These equations are automatically generated by 20SIM as standard C code. They are then converted into a C-MEX function to be integrated with the SIMULINK model.

\subsection{Vehicle dynamics}

This module includes the wheels/tyres, axles, suspensions and body of the vehicle. Models of different complexity can be used, depending on the goals of the simulation. In this work, a single Degree of Freedom (DOF), point mass model, is selected because this paper focuses on the estimation of fuel consumption. The complexity of the simulation modules can be adjusted when other vehicle behaviors such as handling/roll are of interest (Louca et al., 1998). The development of the vehicle dynamics model is given in (Louca et al., 2001) and its key parameter values are given in Table A2 of the Appendix. 


\subsection{Electric sub-systems}

Two electric sub-systems were added for the hybrid powertrain: a DC motor/generator, and a lead-acid battery. Due to the extra weight caused by the electric systems, the hybrid truck was estimated to be $246 \mathrm{~kg}$ heavier than the original vehicle. The power electronics sub-system is assumed to be fast and thus is ignored in the control design. The characteristics of the DC motor/generator and the lead-acid battery are described in the following sub-sections.

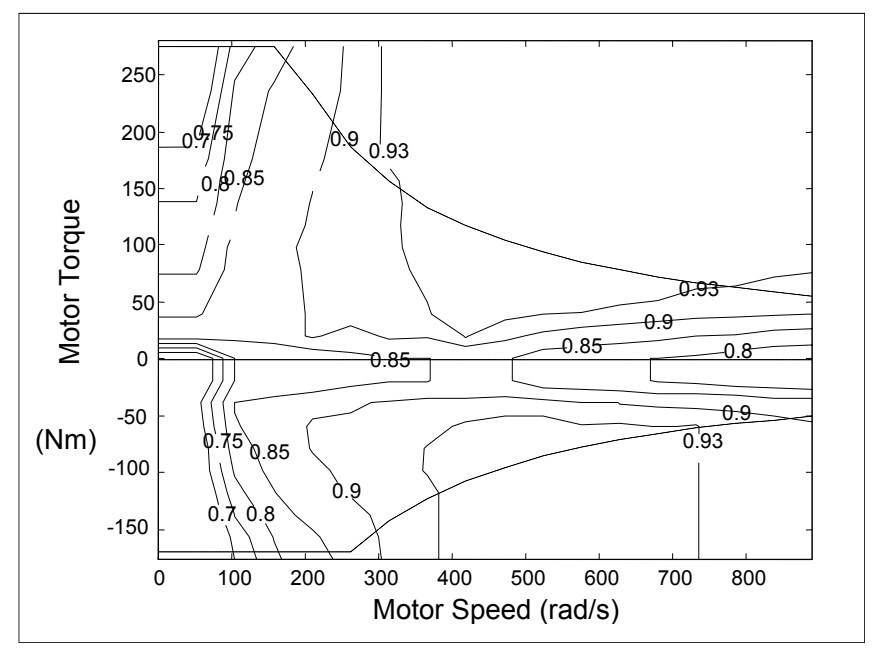

Figure 1 Efficiency map of the DC motor (Burch et al., 1999).

DC-MOTOR/GENERATOR - Because the peak power of the original 8-cylinder engine is $157 \mathrm{~kW}$ while the 6-cylinder engine for the hybrid truck develops approximately $117 \mathrm{~kW}$, a $49 \mathrm{~kW}$ permanent magnet DC motor is selected, so that the hybrid truck has slightly higher peak total power. This motor was selected from the library of motors published in the ADVISOR program (MC-PM49) (Burch et al., 1999). The efficiency/loss data have the form $\eta_{m}=f\left(T_{m}, \omega_{m}\right)$ (see Figure 4). The motor dynamics are approximated by a first-order lag. Due to the battery power and motor torque limit, the final motor dynamics can be determined as follows.

Positive motor torque:

$$
T_{m}=\min \left(T_{m_{-} \text {request }}, T_{m_{-} \max }, T_{m_{-} \text {bat }}\right) \cdot \frac{\lambda_{m}}{s+\lambda_{m}}
$$

Negative motor torque (generator mode):

$$
T_{m}=\max \left(T_{m_{-} \text {request }}, T_{m_{-} \max }, T_{m_{-} \text {bat }}\right) \cdot \frac{\lambda_{m}}{s+\lambda_{m}}
$$

where $T_{m_{-} \text {request }}$ is the requested motor torque, $T_{m_{-} \max }$ is the maximum torque the motor can generate due to the motor constraint, $T_{m_{-} b a t}$ is the maximum motor torque due to battery constraint, $T_{m}$ is the calculated motor torque, and $\lambda_{m}$ characterises the first-order 
lag of the motor dynamics. The load current for the battery can then be calculated from the following equation:

$$
i_{t h}=\left\{\begin{array}{lll}
\frac{\omega_{m} \cdot T_{m} \cdot \eta_{m}}{e_{t h}} & \text { if } & T_{m}<0 \\
\frac{\omega_{m} \cdot T_{m}}{e_{t h} \cdot \eta_{m}} & \text { if } & T_{m}>0
\end{array}\right\}
$$

where $e_{t b}$ is the battery terminal voltage, $\eta_{m}$ is the motor efficiency, and $\omega_{m}$ is the motor speed.

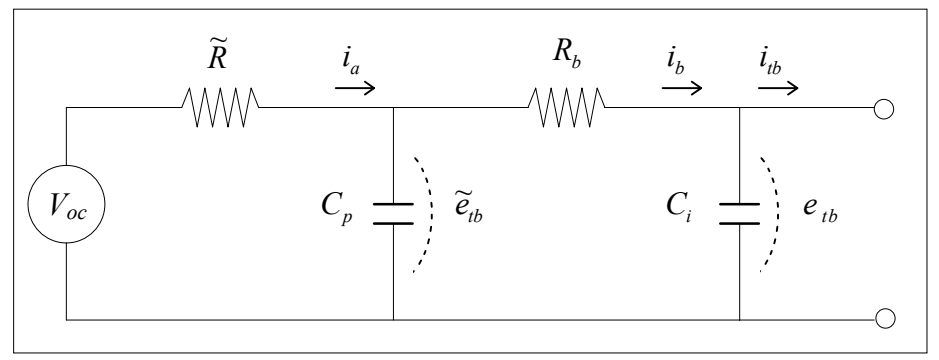

Figure 2 Battery model.

BATTERY - The battery model (Powell and Pilutti, 1994) used is essentially two RC circuits connected in series (see Figure 5). The state-dependent internal resistance $\widetilde{R}$ and the open circuit voltage $V_{o c}$ are lumped representations of a complex chemical process, and are known to be functions of the battery State of Charge (SOC). The terminal ohmic resistance $R_{b}$ and the two capacitances (the polarisation capacitance $C_{p}$ and incipient capacitance $C_{i}$ ) are assumed to be constant. The voltages across the two capacitors and the $\mathrm{SOC}$ are the three state variables of the battery sub-system:

$$
\begin{aligned}
& \frac{d e_{t b}}{d t}=\frac{1}{R_{b} C_{i}}\left(\widetilde{e}_{t b}-e_{t b}-R_{b} i_{t b}\right) \\
& \frac{d \widetilde{e}_{t b}}{d t}=\frac{1}{\widetilde{R} C_{p}}\left(V_{o c}-\left(\frac{R_{b}+\widetilde{R}}{R_{b}}\right) \widetilde{e}_{t b}+\frac{\widetilde{R}}{R_{b}} e_{t b}\right) \\
& \frac{d S O C}{d t}=-\frac{i_{t b}}{q_{m}}
\end{aligned}
$$

where $q_{m}$ is the maximum battery charge. Because the two RC-circuit modes are much faster than the dynamics of SOC, in the simplified model (used for dynamic optimisations) we will only have one state for the battery - the SOC.

In addition to introducing extra dynamics, the battery also plays an important role for the overall fuel economy because of its nonlinear and non-symmetric efficiency characteristics. Figure 6 shows the charging and discharging efficiency of the battery, obtained by reducing the dynamic model to a static one and then computing the instantaneous power efficiency. The procedure for this efficiency calculation can be found in (Wiegman and Vandenput, 1998). It can be seen that the optimal combined 
charging-discharging efficiency is obtained around $\mathrm{SOC}=0.6$. Charging becomes increasingly inefficient at higher SOC, while efficiency of discharging decreases at low SOC.

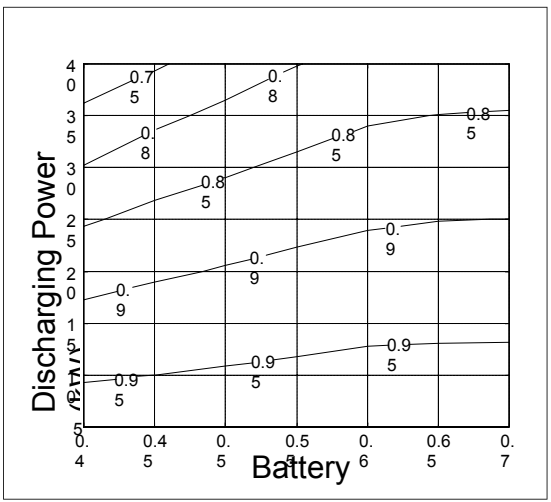

(a)

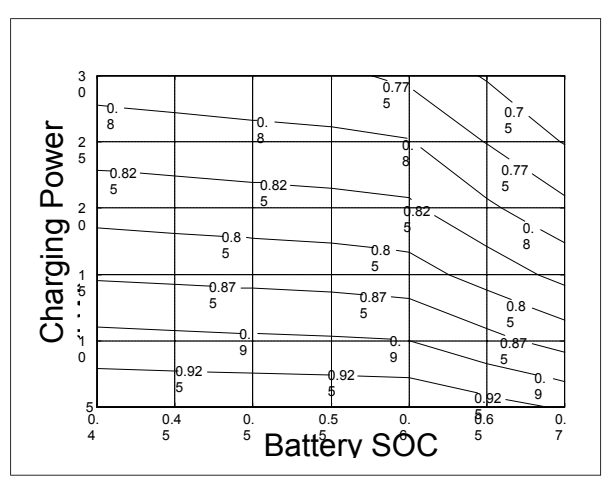

(b)

Figure 3 Efficiency maps of the lead acid battery - (a) discharging efficiency; (b) charging efficiency.

\section{$3 \quad$ Power management algorithm}

The control of complex dynamic systems is usually decomposed into two levels to simplify the control design and analysis (see Figure 7). The main loop controller solves the power management problem, by properly determining the power split between the two power sources, and the transmission gear selection. The design of the main-loop controller is frequently solved using optimal or intelligent approaches. The demanded engine and motor power levels and transmission gears are then achieved by the servolevel controllers. The servo-level control problems are standard regulation or tracking problems, and thus are usually solved by robust control techniques. In this paper, we will only discuss the power management control problem, with the assumption that servolevel controllers are available to achieve the commanded actions. A sub-optimal rulebased algorithm, obtained by learning from a Dynamic Programming (DP) optimisation algorithm will be presented.

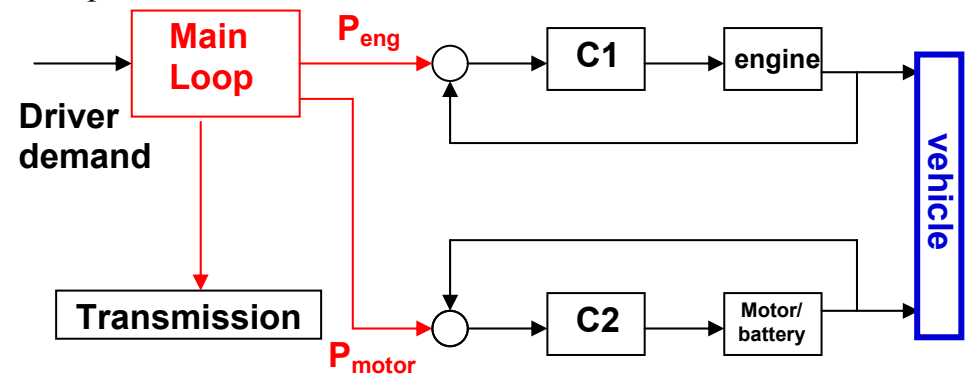

Figure 7 Two-level hybrid vehicle control problem. 
Traditional rule-based algorithms are popular because they take advantage of engineering intuition and experience. However, when the control system is multi-variable and/or multi-objective, as in the case of HEV control, it is usually difficult to come up with rules that capture all the important trade-offs among multiple performance variables. The DP algorithm can help us understand the deficiency of the rules, and subsequently serve as a 'role-model' to construct improved (and more complicated) rules. Using a rule-base algorithm which mimics the optimal actions from the DP approach gives us three distinctive benefits: (i) optimal performance is known from the DP solutions; (ii) the rule-based algorithm is tuned to obtain near-optimal solution, under the predetermined rule structure and number of free parameters; and (iii) the design procedure is re-useable, for other hybrid vehicles, or other performance objectives.

\subsection{Dynamic programming}

As opposed to standard rule-based design approaches, the DP design method relies on a model to compute the best control strategy. The vehicle model can be either analytical or numerical; in other words, it can work with numerical simulation packages such as HEVESIM. In the discrete-time format, the model could be presented in the form $x(k+1)=f(x(k), u(k))$, where $x(k)$ denotes the state variables (vehicle speed, transmission gear position, battery state of charge) of the vehicle at time step $k$, and $u(k)$ are the input signals (engine fuel rate, transmission shift) to the vehicle at time step $k$. The goal of the DP optimisation scheme is to minimise a cost function over a given driving cycle by choosing appropriate control signals $u(k)$. In this paper, the cost function is assumed to consist of only engine fuel consumption. However, it is straightforward to extend the design process to a simultaneous fuel economy-emissions optimisation problem if an emissions model is included. The cost function we used has the following form:

$$
J=f u e l=\sum_{k=0}^{N-1} L(x(k), u(k)) \quad(k g)
$$

where $L$ is the instantaneous fuel consumption rate, and $N$ is the time length of the driving cycle. The driving cycle used in this study is EPA Urban Dynamometer Driving Schedule for Heavy-Duty Vehicles (UDDSHDV) from the ADVISOR drive-cycle library. The optimisation problem is solved under inequality constraints to ensure that the engine speed, SOC, engine torque and motor torque are all within their corresponding bounds. Also, equality constraints are imposed so that the vehicle always follows the specified driving cycle profile. To accelerate the numerical computations, the incremental cost calculated at each grid point for all possible states and control signals is stored in look-up-tables. Details of this look-up-table based DP algorithm have been reported in (Kang et al., 1999) and thus are omitted here. Since the problem formulated above does not impose any penalty on battery energy, the optimisation algorithm tends to first deplete the battery in order to achieve minimal fuel consumption. This charge depletion behaviour will continue until a lower battery SOC is reached. Hence, a final state constraint on SOC needs to be imposed to maintain the energy of the battery and to achieve a fair comparison of fuel economy. A soft terminal constraint on SOC (quadratic penalty function) is added to the cost function as follows: 


$$
J=\sum_{k=0}^{N-1} L(x(k), u(k))+G(x(N))
$$

where $G(x(N))=\alpha\left(\operatorname{SOC}(N)-S O C_{f}\right)^{2}$ represents the penalty associated with the error in the terminal SOC; $S O C_{f}$ is the desired SOC at the final time; and $\alpha$ is a weighting factor.

The DP procedure produces an optimal, time-varying, state-feedback control policy that is stored in a table for each of the quantised states and time stages, i.e. $u^{*}(x(k), k)$; this function is then used as a state feedback controller in the simulations. In addition, DP creates a family of optimal paths for all possible initial conditions. In our case, once the initial SOC is given, the DP algorithm will find an optimal way to bring the final SOC back to the terminal value $\left(S O C_{f}\right)$ while achieving the minimal fuel consumption.

It should be noted that a simplified model (with only three state variables, vehicle speed, SOC and gear number) was generated for the DP algorithm, because the computation time increases exponentially with number of states. The original HEVESIM has 24 states and thus is too complex to be used. Furthermore, the obtained DP solutions are in a time-varying full-state feedback form; hence, using the complex model implies all states must be measured or estimated. Since the control policy determined by the DP algorithm is generated from the simplified model, the control policy needs to be verified on the original complex model. Therefore, the optimal control policy found by DP was applied to the original HE-VESIM model. The terminal SOC constraint was selected as 0.57 , the same as the initial SOC used for the purpose of calculating fuel economy. Dynamic trajectories of the vehicle under the optimal control policy for the UDDSHDV cycle are shown in Figure 8. The difference between the desired vehicle speed (UDDSHDV) and the actual vehicle speed is within $2 \mathrm{mph}$. The SOC trajectory starts at 0.57 and ends around 0.57 with a small quantisation error. Therefore, the DP solutions adequately provide a template to learn from when developing the rule-based control law.

\subsection{Development of sub-optimal rule-based controls}

Due to the fact that the DP algorithm uses future information throughout the whole driving cycle to determine the optimal strategy, it is only optimal for that particular driving cycle, and cannot be implemented as a control law for general, unknown driving conditions. However, it provides good benchmark to learn from, as long as relevant and simple features can be extracted. In this section, an implementable rule-based control strategy incorporating the knowledge extracted from DP results is proposed. The rulebased strategy starts by interpreting the driver pedal motion as a power demand, $P_{d e m}$. When this driver-demanded power $P_{d e m}$ is negative (brake pedal pressed), the motor is used as a generator to recover vehicle kinetic energy. If the vehicle needs to decelerate harder than possible with the 'electric brake', the friction brake will be used. When positive power is requested (gas pedal pressed), either a Power Split Strategy or a Charge-Sustaining Strategy will be applied, depending on the battery state of charge (SOC). Under normal driving conditions, the Power Split Strategy determines the power flow in the hybrid powertrain. When the SOC drops below the lower limit, the controller 
will switch to the Charge-Sustaining Strategy until the SOC reaches a pre-determined upper limit, and then the Power Split Strategy will resume.

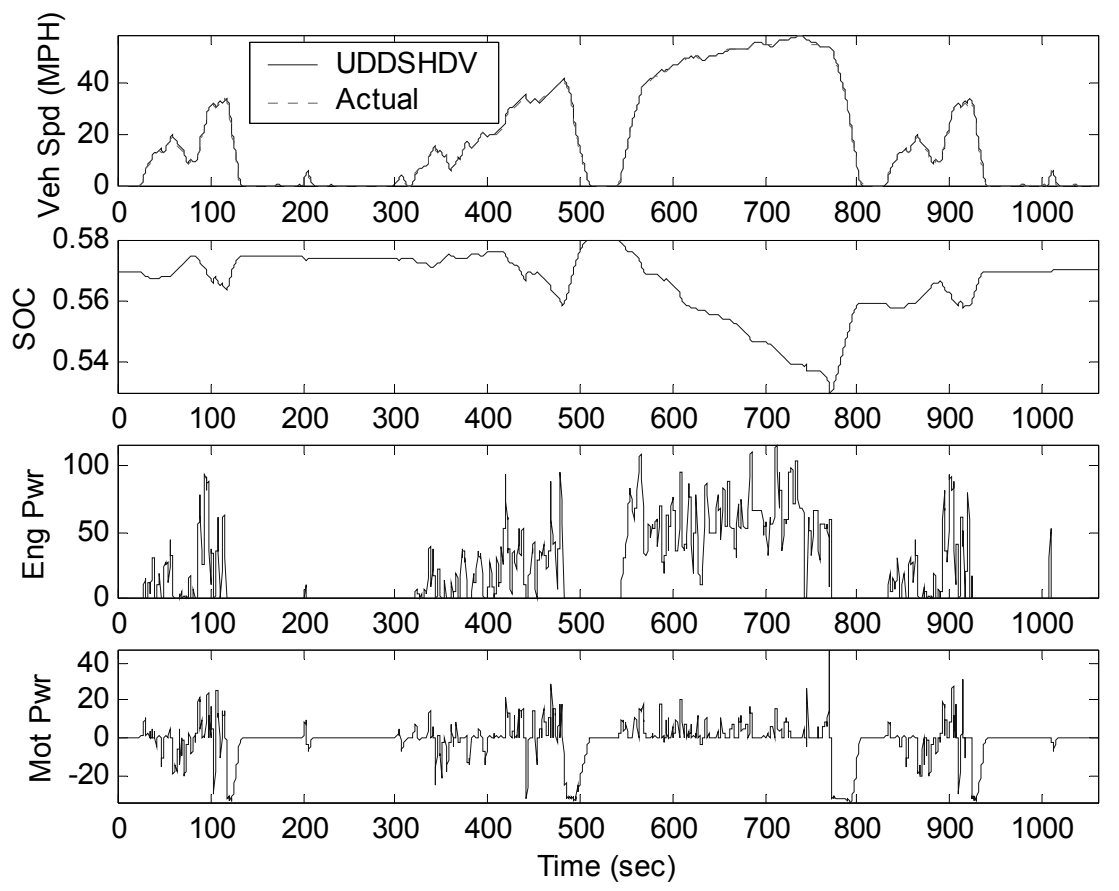

Figure 8 Simulation results by using DP optimal policy over UDDSHDV cycle.

After the structure of the rule-based logic is set, the remaining procedure is to extract rules for the Power Split Strategy, the Charge-Sustaining Strategy, and the Gear Shift Logic. Figure 9 shows a flowchart of the design procedure. The Power Split Strategy and Gear Shift Logic can be learned from the DP approach described in the above section. The DP routine is then repeated with regenerative braking function turned off in order to identify the Charge-Sustaining Strategy. The procedure to extract these strategies from DP is described in the following sub-sections.

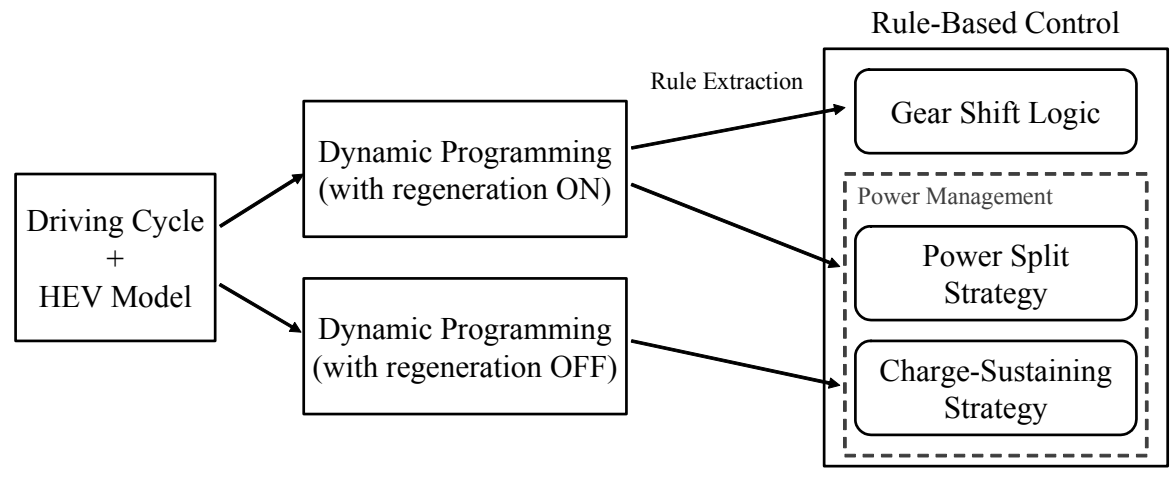

Figure 9 Development of rule-based control strategy. 


\subsubsection{Power split strategy}

In the Power Split Strategy, there are four possible operating modes: motor only mode, engine only mode, hybrid mode (both the engine and motor provide power), and recharge mode (the engine provides additional power to charge the battery). Rules for switching between these modes can be established by examining the DP optimisation results shown in Figure 10.

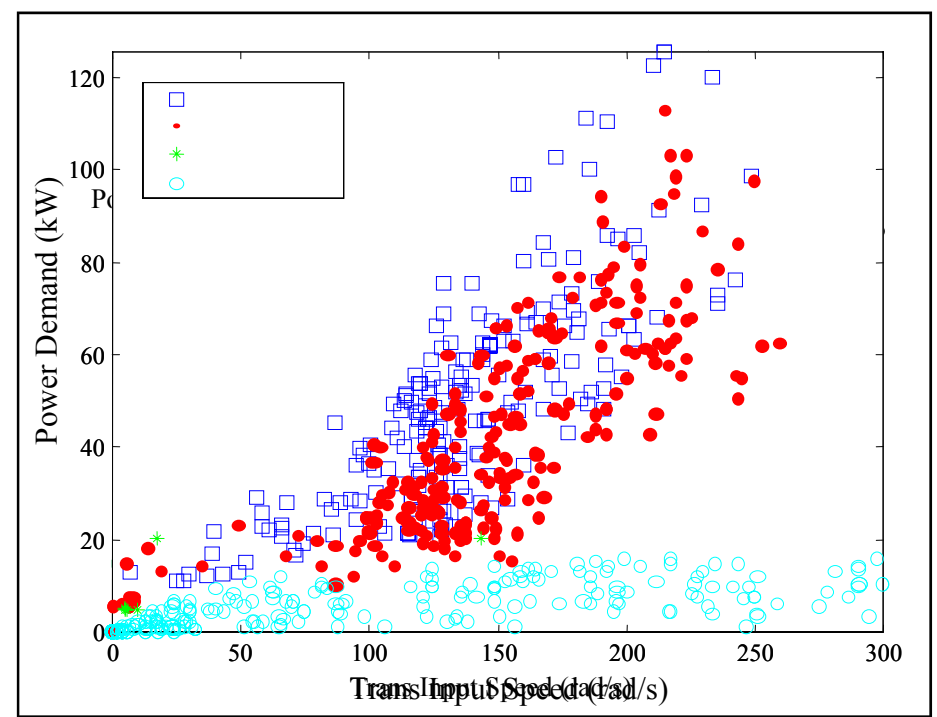

Figure 10 Optimal operating points from DP optimisation over UDDSHDV cycle when $P_{\text {dem }}>0$.

In Figure 10, the $x$-axis denotes the transmission input shaft speed and $y$-axis denotes the power demand. The following observations can be made:

- Use motor-only when power demand is less than $15 \mathrm{~kW}$.

- In region A, DP chooses to operate in the power-split mode.

- Recharging rarely happens.

The rare occurrence of recharging events implies that, under the current vehicle configuration and driving cycle, it is not efficient to use engine power to charge the battery, even when increasing the engine's power would move its operation to a more efficient region. As a result, we assume there is no recharging during power split control, other than regeneration, and thus recharge by the engine will only occur when SOC is too low, which will be described in the charge-sustaining strategy in Section 3.2.3. The distribution between the two prime movers in the hybrid mode is determined next. We will extract from the DP solution an optimal motor power level in the hybrid mode, and then determine the engine power demand by subtracting the motor power from the driver power demand. Clearly, optimal motor power may depend on many variables such as wheel speed, engine speed, power demand, SOC, gear ratio, etc. For this reason, a 
regression-based program was first used to assess which of these variables were the dominant factors in determining the motor power. We found that power demand, engine speed, and transmission input speed were the most important factors. Motor power, as determined by the DP algorithm, was then used as the desired output for a Neural Network (NN), using two hidden layers with 3 and 1 neurons, respectively. After training the $\mathrm{NN}$, it can then be used in real-time as an implementable 'look-up table' to determine the proper motor power. The inputs to this $\mathrm{NN}$ are the three variables listed above. The basic logic of this sub-optimal power split control is summarised in Table 2.

Table 2 Basic logic rules of power split strategy.

If $P_{d e m} \leq 15 \mathrm{~kW}, P_{m}=P_{d e m} \quad P_{e}=0$

Else If Region A, $P_{m}=$ Nnet $_{1}\left(P_{\text {dem }}, \omega_{\text {trans }}, \omega_{\text {eng }}\right) \quad P_{e}=P_{\text {dem }}-P_{m}$

If Region B, $P_{m}=0 \quad P_{e}=P_{\text {dem }}$,

If $P_{e}>P_{e_{-} \max }, P_{e}=P_{e_{-} \max } \quad P_{m}=P_{d e m}-P_{e}$

\subsubsection{Gear shift logic}

Determining the transmission shift schedule is crucial to improving the efficient operation of the internal combustion engine. In the DP scheme, transmission gear shift is one of the control inputs to the system. It is important to find out how the DP solution chooses the gear position to improve fuel economy. From the optimisation results, the gear operation points are expressed on the engine power demand vs. wheel speed plot (Figure 11).

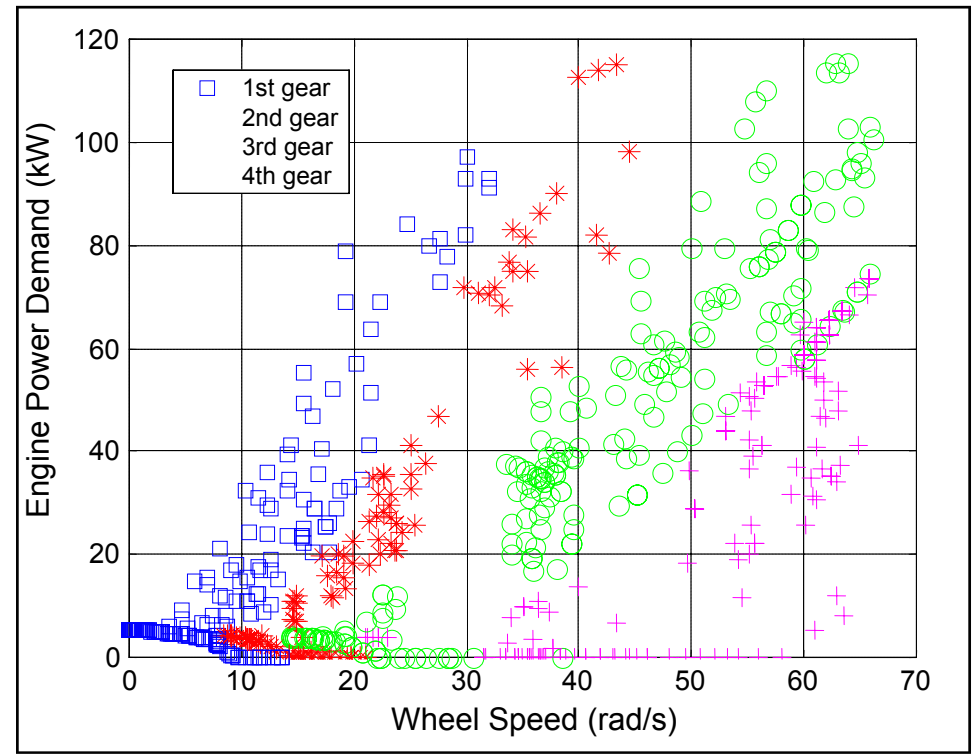

Figure 11 Transmission gear selection from DP algorithm when $P_{\text {dem }}>0$. 
It can be seen that the optimal gear positions are separated into four regions, and the boundary between two adjacent regions seems to represent better gear shifting thresholds. After adding a hysteresis function to the shifting thresholds, a new gear shift map determining when an upshift or downshift event occurs was developed. It should be mentioned that the optimal gear shift map for minimum fuel consumption can also be constructed through static optimisation. Given an engine power and wheel speed, the best gear position for minimum fuel consumption can be chosen based on the steady-state engine fuel consumption map. It was found that the steady-state gear map nearly coincides with Figure 11. This is not surprising since the electric motor is positioned after the transmission. Therefore, the engine efficiency will dominate the formulation of gear shifting policy. For a pre-transmission hybrid configuration, it will be harder to obtain optimal shift map using traditional methods.

\subsubsection{Charge-sustaining strategy}

The Power Split Strategy described above does not always maintain the battery SOC within the desired operating range. An additional rule for charging the battery with the engine should be developed to prevent battery depletion. However, the strategy for sustaining the SOC still needs to be obtained in a near-optimal manner. In the rule-based control algorithm, a thermostat-like charge sustaining strategy is used (Bowles, 1999). The recharging mode will turn on only if the battery SOC falls below the lower limit and continues until the SOC reaches a predetermined level. However, requiring the engine to provide a constant recharging power level, although commonly used because of its simplicity, is not necessarily the most efficient way to recharge the battery. To improve the overall fuel efficiency further, the questions 'when to recharge' and 'at what rate' need to be answered. Hence, specially designed simulation runs were performed to generate more recharging actions. First, the regenerative braking function was turned off in the DP routine. In other words, all the braking power was supplied by the friction braking and hence there was no 'free' energy available from the regenerative braking. Furthermore, the initial SOC was set at 0.52 for the purpose of simulating the situation that SOC is too low and the battery needs to be recharged. The simulation result is shown in Figure . Note that the results obtained represent the optimal policy under the condition that the battery SOC has to be recharged from 0.52 to 0.57 using engine power. Note also that negative motor power now represents the recharging power supplied by the engine since there is no regenerative braking.

Several rules were extracted from the optimisation result as follows:

- Recharging happens only when wheel speed is greater than $10 \mathrm{rad} / \mathrm{s}$ for better generator efficiency.

- $\quad$ Recharging power is usually lower than $15 \mathrm{~kW}$ for better battery charging efficiency.

- The electric motor is the only power source to drive the vehicle when power demand is less than $8 \mathrm{~kW}$ so as to avoid low engine efficiency. 

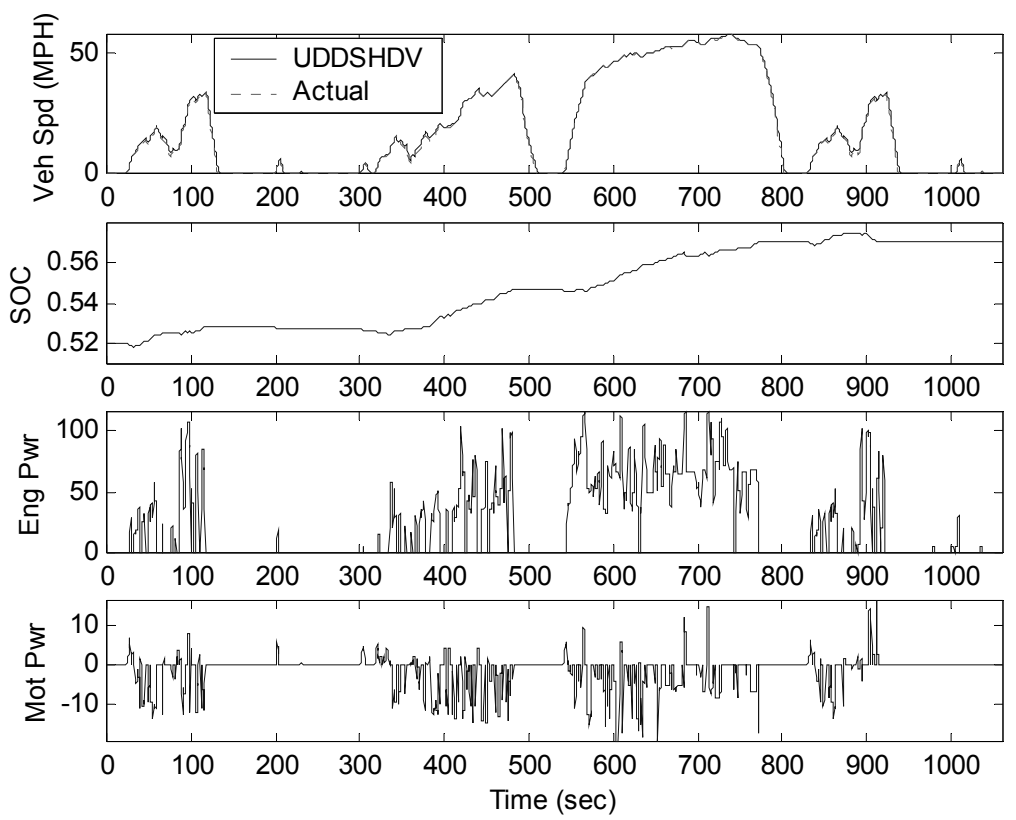

Figure 12 Recharging by using DP optimal policy over UDDSHDV cycle (no regenerative braking).

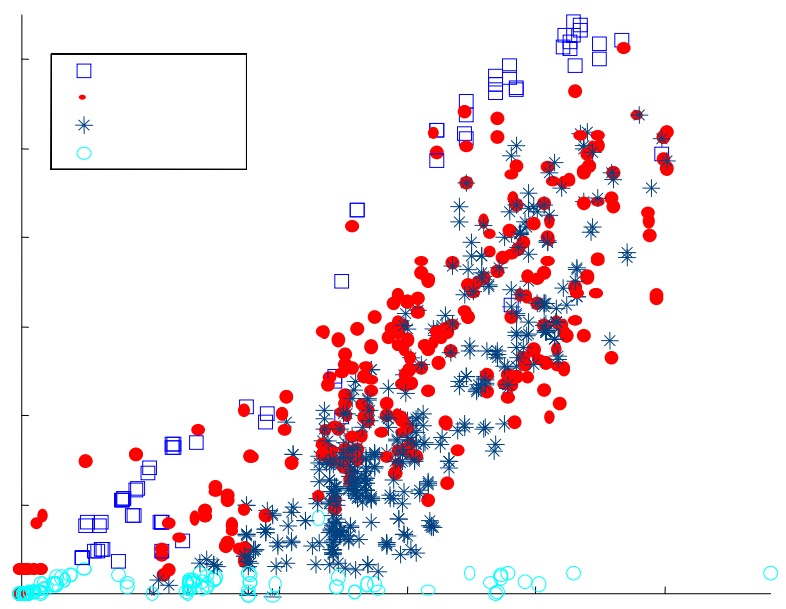

Figure 13 Optimal operating points from DP (engine recharging scenario) over UDDSHDV cycle, $P_{\text {dem }}>0$.

Additional rules can be constructed by analyzing the DP behaviour shown in Figure 13. A threshold line is drawn to divide the plot into two regions. There are few recharging events in Region $\mathrm{C}$, and most of the recharging events happen in region $\mathrm{D}$. 
We focus on the recharging data in region $\mathrm{D}$ to determine a function for optimal recharging power, using the method described above. A regression program was first used to find which factors should be used to build the model, and then a NN was used to fit the function. The basic logic of this recharging control is summarised in Table 2. Combining these rules with the rules developed in Section 3.2.1 provides complete power management of the hybrid propulsion system under any conditions.

Table 2 Basic logic rules of charge-sustaining strategy.

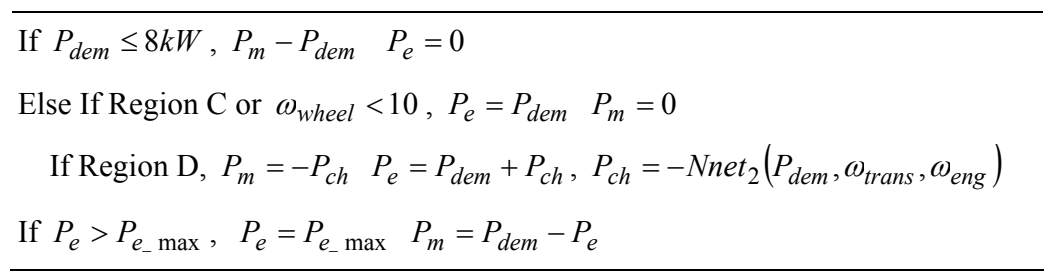

\section{Simulation results}

The hybrid control strategy developed above was integrated into the HE-VESIM model. Two test scenarios were performed to evaluate its effectiveness. The performance of the hybrid vehicle under a hard acceleration from 0 to $60 \mathrm{mph}$ is first assessed. This manoeuvre serves two purposes: First, the launch-drivability of the hybrid vehicle is compared to the original truck. Secondly, it is a common scenario to assess the behaviour of the hybrid control algorithm. By examining the behaviour of the engine, the motor and transmission, we can assess whether the control algorithm behaves reasonably in this simple but important manoeuvre. This test, however, is inadequate in assessing fuel economy because it is too short and is not representative of real driving conditions. We subsequently test the behaviour of the hybrid electric truck over the UDDSHDV cycle, a 17-minute driving schedule in order to evaluate its fuel economy performance.

ACCELERATION $0-60 \mathrm{mph}$ - The 0 to $60 \mathrm{mph}$ acceleration test was simulated to verify that the parallel hybrid with the downsized engine plus the induction motor retains or exceeds the acceleration performance of the conventional vehicle. In this simulation, wide open throttle is kicked in at $t=5 \mathrm{sec}$. The vehicle speed profiles are shown in Figure 14. The hybrid truck (HE-VESIM) achieved a speed of $60 \mathrm{mph}$ slightly earlier than the conventional truck (VESIM), primarily due to the high electric motor torque at low speeds (see Figure 15); the latter torque is especially beneficial because it compensates for the slower response of the diesel engine due to the turbo lag. Therefore, the hybrid powertrain provides higher acceleration at launch. The engine speed history shown in Figure 15 illustrates the turbo lag period during the 5 to 10 seconds interval, as well as fluctuations occurring at each gear shift. Figure 15 also presents the cumulative fuel consumption of the hybrid and the original vehicles. Obviously, the downsized engine consumes less fuel, but it should be kept in mind that part of the energy comes from the battery. The real comparison of fuel consumption should be made over long driving cycles such as the UDDSHDV cycle shown below. 


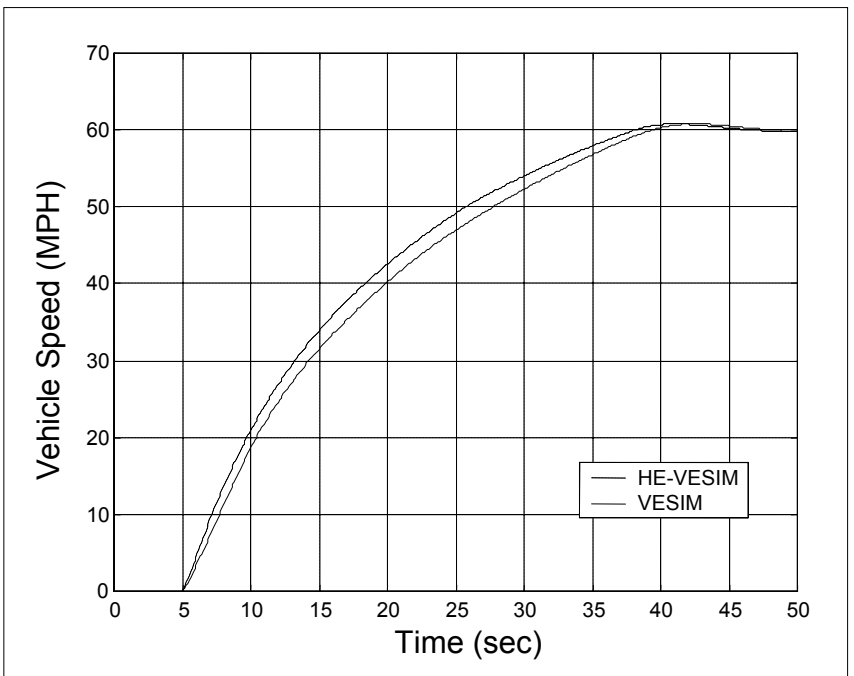

Figure 14 Speed profile comparison of conventional vs. hybrid truck during 0-60 mph acceleration.
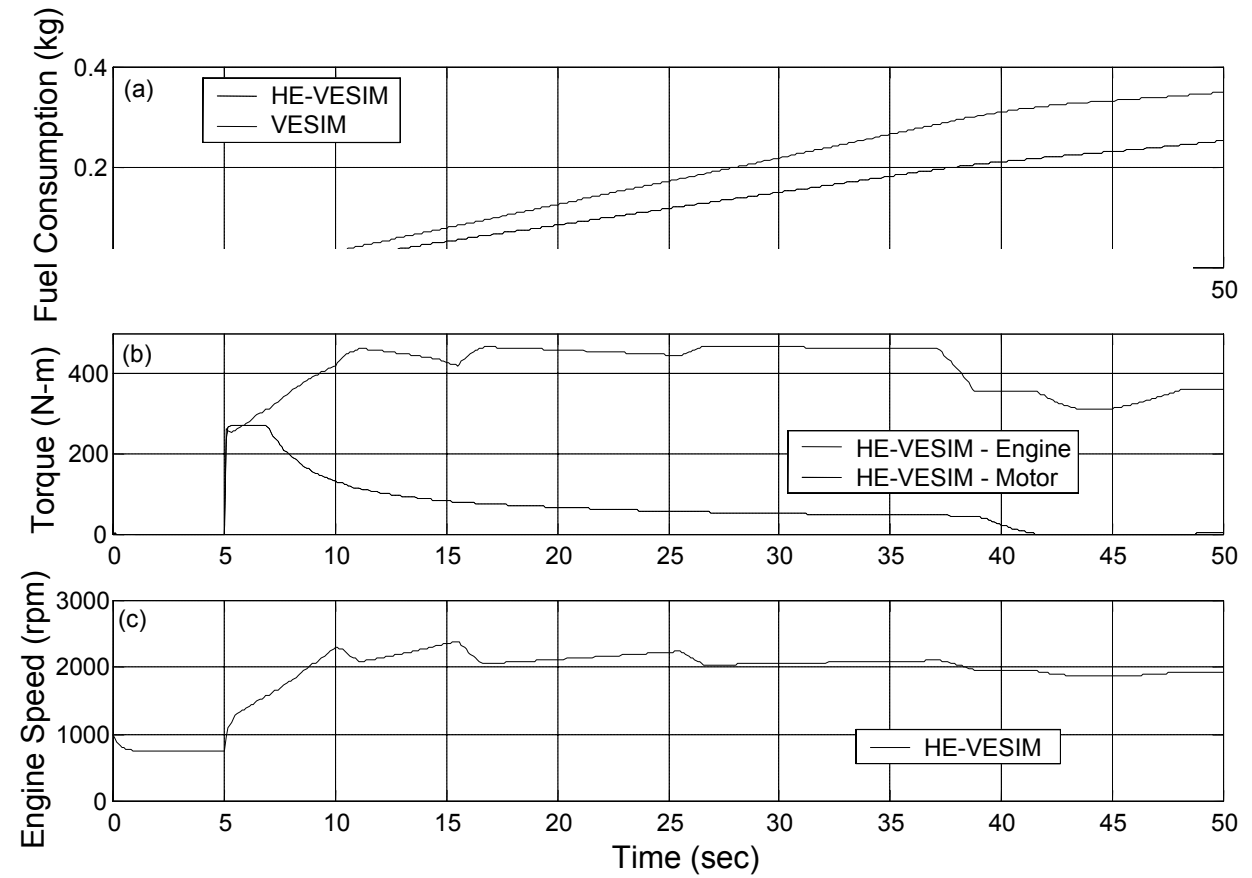

Figure 15 0-60 mph acceleration: conventional truck (VESIM) vs. the hybrid truck (HE-VESIM).

FUEL ECONOMY OVER A DRIVING CYCLE - The Urban Dynamometer Driving Schedule for Heavy-Duty Vehicles (UDDSHDV) was used to evaluate the fuel economy 
in this work. The fuel economy results over the complete urban driving schedule, obtained for our rule-based control algorithm, are compared with a conventional diesel engine truck in Table 4 These results are obtained for the charge sustaining strategy, with the SOC at the end of the cycle being the same as it was at the beginning. It can be seen that the fuel economy improvement (over the conventional truck) is about $28 \%$ for the DP algorithm and 24\% for the DP-trained rule-based algorithm. Knowing that the DP algorithm is the absolute optimal that can be achieved, we determined the performance of the improved rule based algorithm to be quite satisfactory.

Table 3 Fuel economy comparison over UDDSHDV cycle

\begin{tabular}{lc}
\hline & Fuel economy (MPG) \\
Conventional & 10.63 \\
Improved rule-based control & 13.24 \\
DP & 13.63 \\
\hline
\end{tabular}

\section{Summary and conclusions}

In this paper, we present the development of a forward-looking, parallel, hybrid electric vehicle simulation, and its application for the evaluation of power management strategies aimed at maximising fuel economy. The tool is based on a previously validated simulator of conventional engine-vehicle systems that includes modules of the diesel engine, driveline and vehicle dynamics of appropriate fidelity. The electric components were developed and integrated into the simulation with the motor/generator located after the transmission and linked to the output shaft via an electro-mechanical coupling. The diesel engine is downsized since the electric motor is able to provide assistance during the high power demand operation. Two power management algorithms were analysed in this paper - a DP algorithm and a rule-based algorithm. A key idea shown in this paper is using the DP results to obtain an improved rule-based control law. The control strategy aims at maximising fuel economy by coordinating the operation of engine and motor/generator, while sustaining the battery state of charge.

The acceleration performance of the Class VI HEV truck was shown to be comparable to its conventional counterpart. The favourable torque characteristic of the electric motor more than compensates for the delay in the diesel engine's response caused by turbo lag. Simulations over a complete urban driving cycle for heavy-duty vehicles show that the fuel economy could be improved by up to $28 \%$ over the traditional (non-hybrid) truck. While the DP algorithm achieves the highest overall fuel economy, it is not implementable in real driving. Nonetheless, analysing the DP results provides insight into how the fuel economy can be improved by coordinating multiple power sources, which may not be easily captured by simple engineering intuition. It was found that fuel economy improvements were derived mainly from optimising the gear-shifting policy and discharging/charging schedule, and relieving the engine load through more efficient motor/battery operation. Hence, an implementable, sub-optimal rule-based control strategy was developed for real driving application and its fuel economy was found to approach that of the optimal result. 


\section{Acknowledgements}

The authors would like to acknowledge the technical and financial support of the Automotive Research Center (ARC) by the National Automotive Center (NAC) located within the US Army Tank-Automotive Research, Development and Engineering Center (TARDEC). The ARC is a US Army Center of Excellence for Automotive Research at the University of Michigan, currently in partnership with University of AlaskaFairbanks, Clemson University, University of Iowa, Oakland University, University of Tennessee, Wayne State University, and University of Wisconsin-Madison.

\section{References}

20SIM (1999) 20SIM Pro Users' Manual, The University of Twente - Controllab Products B.V. Enschede, The Netherlands.

Assanis, D., Delagrammatikas, G., Fellini, R., Filipi, Z., Liedtke, J., Michelena, N., Papalambros, P., Reyes, D., Rosenbaum, D., Sales, A. and Sasena, M. (1999) 'An optimization approach to hybrid electric propulsion system design', Mechanics of Structures and Machines, Vol. 27, No. 4, pp. $393-421$.

Assanis, D.N., Filipi, Z.S., Gravante, S., Grohnke, D., Gui, X., Louca, L.S., Rideout, G.D., Stein, J.L. and Wang, Y. (2000) 'Validation and use of SIMULINK integrated, high fidelity, engine-invehicle simulation of the international class VI truck', SAE Paper 2000-01-0288, SAE 2000 World Congress.

Assanis, D.N. and Heywood, J.B. (1986) 'Development and use of a computer simulation of the turbocompounded diesel system for engine performance and component heat transfer studies,' SAE Paper 860329.

Baumann, B.M., Washington, G., Glenn, B.C. and Rizzoni, G. (2000) 'Mechatronic design and control of hybrid electric vehicles,' IEEE/ASME Transactions on Mechatronics, Vol. 5, No. 1, pp. 58-72.

Bowles, P. D. (1999) 'Modeling and energy management for a parallel hybrid electric vehicle (PHEV) with continuously variable transmission (CVT)', MS thesis, University of Michigan, Ann Arbor, MI.

Brahma, A., Guezennec, Y. and Rizzoni, G. (2000) 'Dynamic optimization of mechanical/electrical power flow in parallel hybrid electric vehicles' Proceedings of AVEC 2000, 5th Int'l Symposium on Advanced Vehicle Control, Ann Arbor, MI.

Burch, Steve and Cuddy, Matt et al. (1999) 'ADVISOR 2.1 documentation,' National Renewable Energy Laboratory, March.

Farrall, S.D. and Jones, R.P. (1993) 'Energy management in an automotive electric/hear engine hybrid powertrain using fuzzy decision making.' Proceedings of the 1993 International Symposium on Intelligent Control, Chicago, IL.

Johnson, V.H., Wipke, K.B. and Rausen, D.J. (2000) 'HEV control strategy for real-time optimization of fuel economy and emissions,' Proceedings of the Future Car Congress, April 2000, SAE paper 2000-01-1543.

Kang, J.-M., Kolmanovsky, I. and Grizzle J.W. (1999) 'Approximate dynamic programming solutions for lean burn engine aftertreatment,' Proceeding of the IEEE Conference on Decision and Control, Phoenix, AZ, December 7-10.

Karnopp, D.C., Margolis, D.L., and Rosenberg, R.C. (1990) System Dynamics: A Unified Approach, Wiley-Interscience, New York, New York. 
Kim, C., NamGoong, E. and Lee, S. (1999) 'Fuel economy optimization for parallel hybrid vehicles with CVT.' SAE Paper No. 1999-01-1148.

Liu, H., Chalhoub, N.G. and Henein, N. (1997) 'Simulation of a single cylinder diesel engine under cold start conditions using SIMULINK', Proceedings of ASME-ICE Spring Technical Conference, Fort Collins, CO, Vol. 28-1, April 27-30.

Louca, L.S., Stein, J.L. and Hulbert, G.M. (1998) 'A physical-based model reduction metric with an application to vehicle dynamics', The 4th IFAC Nonlinear Control Systems Design Symposium (NOLCOS 98), Enschede, The Netherlands.

Louca, L.S., Stein, J.L. and Rideout, D.G. (2001) 'Generating proper integrated dynamic models for vehicle mobility using a bond graph formulation', Proceedings of the 2001 International Conference on Bond Graph Modeling, The Society for Computer Simulation, Phoenix, AZ, January.

Paganelli, G., Ercole, G., Brahma, A., Guezennec, Y. and Rizzoni, G. (2000) 'A general formulation for the instantaneous control of the power split in charge-sustaining hybrid electric vehicles.' Proceedings of AVEC 2000, 5th Int'l Symposium on Advanced Vehicle Control, Ann Arbor, MI.

Powell, B.K. and Pilutti, T.E. (1994) 'A range extender hybrid electric vehicle dynamic model', Proceedings of the $33^{\text {rd }}$ IEEE Conference on Decision and Control, Lake Buena Vista, FL, December.

Rosenberg, R.C. and Karnopp, D.C. (1983) Introduction to Physical System Dynamics, McGrawHill, New York, New York.

Wiegman, H.L.N. and Vandenput, A.J.A. (1998) 'Battery state control techniques for charge sustaining applications,' SAE Paper 981129.

\section{Appendix}

Table A1 Drivetrain specifications.

\begin{tabular}{lc}
\hline Torque converter - Turbine inertia $\left[\mathrm{kg}^{*} \mathrm{~m}^{2}\right]$ & 0.068 \\
Transmission - 1st gear churning losses coeff. R11 & 0.0192 \\
Transmission - 2nd gear churning losses coeff. R12 & 0.015 \\
Transmission - 3rd gear churning losses coeff.R13 & 0.031 \\
Transmission - 4th gear churning losses coeff.R14 & 0.0367 \\
Transmission - 1st gear churning losses coeff.R21 & $1.361 * 10^{-5}$ \\
Transmission - 2nd gear churning losses coeff.R22 & $5.719^{*} 10^{-6}$ \\
Transmission - 3rd gear churning losses coeff.R23 & $-3.189 * 10^{-5}$ \\
Transmission - 4th gear churning losses coeff.R24 & $-4.177 * 10^{-5}$ \\
Transmission - 1st gear ratio [-] & 3.45 \\
Transmission - 2nd gear ratio [-] & 2.24 \\
Transmission - 3rd gear ratio [-] & 1.41 \\
Transmission - 4th gear ratio [-] & 1.00 \\
Transmission - 1st gear ratio [-] FOR HYBRID & 2.59 \\
Transmission - 2nd gear ratio [-] FOR HYBRID & 1.68 \\
\hline
\end{tabular}




\begin{tabular}{lc}
\hline Transmission - 3rd gear ratio [-] FOR HYBRID & 1.06 \\
Transmission - 4th gear ratio [-] FOR HYBRID & 0.75 \\
Transmission - Fluid charging pump loss [N*m] & -6.12 \\
Transmission - 1st Gear efficiency [-] & 0.9893 \\
Transmission - 2nd Gear efficiency [-] & 0.966 \\
Transmission - 3rd Gear efficiency [-] & 0.9957 \\
Transmission - 4th Gear efficiency [-] & 1.0 \\
Propshafts/Differential - Axle churning loss coeff.R0 & 8.34 \\
Propshafts/Differential - Axle churning loss coeff.R1 & 0.04087 \\
Propshafts/Differential - Differential drive ratio [-] & 3.21 \\
Propshafts/Differential - Differential efficiency [-] & 0.96 \\
\hline
\end{tabular}

Table A2 Vehicle dynamics specifications.

\begin{tabular}{|c|c|}
\hline CG location from front axle [-] & 0.61875 \\
\hline Sprung mass $[\mathrm{kg}]$ & 6581.6 \\
\hline Unsprung mass rear $[\mathrm{kg}]$ & 430.9 \\
\hline Unsprung mass front $[\mathrm{kg}]$ & 244.9 \\
\hline Longitudinal - Wheel inertia $\left[\mathrm{kg}^{*} \mathrm{~m}^{2}\right]$ & 18.755 \\
\hline Longitudinal - Break viscous damping $[\mathrm{N} * \mathrm{~m} * \mathrm{~s} / \mathrm{rad}]$ & 100.0 \\
\hline Longitudinal - Break coulomb damping $[\mathrm{N} * \mathrm{~m}]$ & 0.0 \\
\hline Longitudinal - Wheel radius [m] & 0.4131 \\
\hline Longitudinal - Tire pressure [psi] & 115.0 \\
\hline Longitudinal - Number of tires on rear axle [-] & 4.0 \\
\hline Longitudinal - Wheel bearing damping $\left[\mathrm{N}^{*} \mathrm{~m} * \mathrm{~s} / \mathrm{rad}\right]$ & 3.0 \\
\hline Longitudinal - Road/tire friction coefficient [-] & 0.7 \\
\hline Longitudinal- Aerodynamic drag coefficient & 2.081 \\
\hline Vertical - Rear suspension compliance $[\mathrm{m} / \mathrm{N}]$ & $6.3446110^{-7}$ \\
\hline Vertical - Rear tire compliance $[\mathrm{m} / \mathrm{N}]$ & $2.9740310^{-7}$ \\
\hline Vertical - Rear suspension damping [ $\mathrm{N} * \mathrm{~s} / \mathrm{m}]$ & 7000.0 \\
\hline Vertical - Rear tire damping $\left[\mathrm{N}^{*} \mathrm{~s} / \mathrm{m}\right]$ & 2000.0 \\
\hline
\end{tabular}

